\title{
基于视觉信息融合的熔丝成形制造路径在线识别
}

\author{
常树鹤 ${ }^{1}$ 张昊宇 ${ }^{1}$ 许海鹰 ${ }^{2}$ 洪宇翔 $^{1}$ 王 力 $^{1}$ 都 东 ${ }^{1}$ \\ (1. 清华大学机械工程系 北京 100084 ; \\ 2. 北京航空制造工程研究院 北京 100024)
}

\begin{abstract}
摘要: 在能源装备制造领域大型构件多层多道电弧焊接和航空制造领域重要构件电子束熔丝沉积增材制造过程中, 迫切需要 路径在线识别及实时自动导引技术。然而在熔丝制造过程中，相邻焊道之间的几何差异很小，传统的结构光焊缝识别技术无 法适应。为解决该问题, 针对多层多道电弧焊和电子束熔丝沉积增材制造两种场景, 分别设计基于双侧定向光影与结构光信 息融合的视觉检测装置, 并进行成形试验。试验结果表明, 将双侧定向光源单独点亮时获得的焊缝灰度图像作差时, 焊道边 界附近呈现明显的 “高灰度-低灰度-高灰度” 的过渡区域。时域融合处理可以有效消除弧光、飞浌的干扰。提出的定向光影 视觉信息与结构光传感信息实时融合处理方法, 较之已有基于线结构光信息的焊道识别和自动跟踪系统在某些场合具有更强 适应性, 为保证成形质量和提高生产效率提供了新的技术途径。
\end{abstract}

关键词: 多层多道电弧焊接; 熔丝沉积增材制造; 路径在线识别; 视觉检测; 信息融合

中图分类号: TG156

\section{Online Path Recognition for Additive Manufacturing and Arc Welding with Filler Wire Based on Multi-vision Information Fusion}

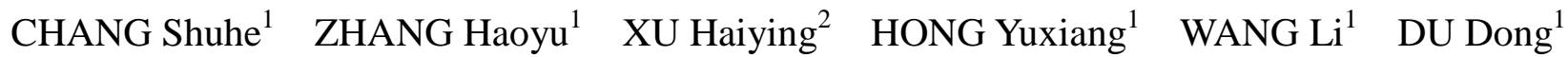

(1. Department of Mechanical Engineering, Tsinghua University, Beijing 100084;

2. Beijing Aeronautical Manufacturing Technology Research Institute, Beijing 100024)

\begin{abstract}
In the manufacturing process of multi-layer/multi-pass weld of large-scale components in the field of energy equipment and electron beam freeform fabrication in the field of aerospace manufacturing, there is an urgent need for online recognition and real-time automatic guidance technology. But the geometrical differences is very small between adjacent weld passes which makes it difficult to detect the weld position using traditional methods such as structured light. A method of real-time fusion processing of directional and structured light information is developed to solve this problem, which can be used in multi-layer/multi-pass weld and electron beam freeform fabrication. Corresponding visual detection devices are also designed. The result shows that there is a "High-Low-High" distribution of brightness between adjacent passes when using differential image. The act of fusing adjacent images greatly reduces the influence of the spatters and arc light. Compared with the existing weld path recognition and automatic tracking system based on structured light method, it has stronger adaptability to ensure the processing quality. This method also provides a new technical approach to improve the productivity.
\end{abstract}

Key words: multi-layer/multi pass arc welding; wire feed additive manufacturing; in-situ seam tracking; information fusion; visual detection

\section{0 前言}

作为一类重要的先进制造技术, 熔丝成形制造 得到了很多研究者的关注, 其包括熔丝沉积增材制 造和熔化极送丝焊接。DING 等 ${ }^{[1]}$ 从基本原理、成

* 国家自然科学基金(51875309)、国家工信部基金(xx-079)和国家重点研发 计划(2017YFB1103100)资助项目。20180926 收到初稿, 20190306 收到 修改稿
形质量、成形精度等方面对基于电子束、激光和电 弧热源的熔丝沉积增材制造方法进行了对比, 并指 出过程监控技术对于提高熔丝沉积增材制造零件 质量有着非常重要的作用。TAMINGER 等 ${ }^{[2]}$ 开发 了一套电子束熔丝沉积增材制造设备, 其沉积效率 可达 $2500 \mathrm{~cm}^{3} / \mathrm{h}$ 。GOCKEL 等 ${ }^{[3]}$ 提出了一种电子 束熔丝沉积工艺下的凝固组织控制技术, 可以大幅 提高成形件性能。ZHANG 等 ${ }^{[4]}$ 利用有限元法对电 子束异种金属填丝焊接进行了研究, 分析结果表明 
填丝焊接可明显降低长度方向上的残余应力。 WANG 等 ${ }^{[5]}$ 研究了电子束电流对异种金属填丝焊 接的影响, 指出其影响机制主要为熔深。NIE 等 ${ }^{[6]}$ 开发了一套激光熔丝增材制造设备, 沉积效率可达 $3.46 \mathrm{~kg} / \mathrm{h}$, 并且所需激光器功率小于 $10 \mathrm{~kW}$ 。 $\mathrm{DEMIR}^{[7]}$ 基于微秒激光的增材制造设备, 并成功沉 积出了薄壁和块体零件。LIU 等 ${ }^{[8]}$ 利用光谱仪对激 光填丝焊中的激光诱导等离子体进行监控, 结果表 明当热丝电压大于 $9 \mathrm{~V}$ 时, 会激发大量飞溅, 对等 离子体产生扰动, 严重影响焊接稳定性。WANG 等 ${ }^{[9]}$ 基于电弧增材制造技术成功沉积出了单壁零 件, 并对其显微组织进行了分析, 其指出发展电弧 增材制造的缺陷无损检测技术对于此技术应用于 航空领域非常有必要。

本文研究对象主要是能源装备制造领域大型构 件多层多道电弧焊接和航空制造领域重要构件电子 束熔丝沉积增材制造过程。尽管在熔丝成形制造的 工业化生产应用中都要开展工艺规划, 包括事先进 行的离线路径规划, 但由于实际成形过程存在熔滴 过渡、工件基体导热等诸多复杂因素影响, 为保证 成形质量和生产效率，还必须研究开发成形过程路 径在线识别和实时自动导引技术。

视觉信息由于其信息量大、抗干扰能力强等优 点, 在焊前坡口检测、焊中焊缝跟踪、焊后质量 检测等场合中具有广泛应用 ${ }^{[10-12]}$ 。邹炎送等 ${ }^{[13]}$ 提 出了一种基于概率连续模型的焊缝跟踪算法, 能 够抵抗较强的外界干扰。ZENG 等 ${ }^{[14-17]}$ 基于定向光 影和结构光信息, 实现了对窄间隙焊缝的检测、 示教和实时跟踪。SHAO 等 ${ }^{[18-20]}$ 基于 $X$ 射线成像 技术实现了对焊缝缺陷的无损检测。ZOU 等 ${ }^{[21-25]}$ 利用卡尔曼滤波、对象特征分析、焊缝纹理特征、 图像色彩信息与多信息融合技术，采用多种不同 方法实现了对焊缝的视觉识别。本文基于以往在 视觉检测方面的研究工作积累, 提出采用定向光 影视觉信息与结构光传感信息实时融合处理的新 方法, 探索解决多层多道熔丝成形路径快速可靠 识别的有效途径。

\section{1 视觉检测试验系统构建}

本文研究工作分别构建了电弧熔丝多层多道焊 接试验装置和电子束熔丝沉积增材制造试验装置。

电弧熔丝多层多道焊接试验装置如图 1 所示, 包括: 熔化极弧焊系统, 多自由度焊接机器人+转台 系统，大坡口多层多道焊接合金钢工件，线结构光 源, 左右对称的定向光源, 用于产生同步触发信号
波形的可编程逻辑控制器 (Programmable logic controller, PLC), 千兆以太网工业相机，计算机图像 处理系统等。

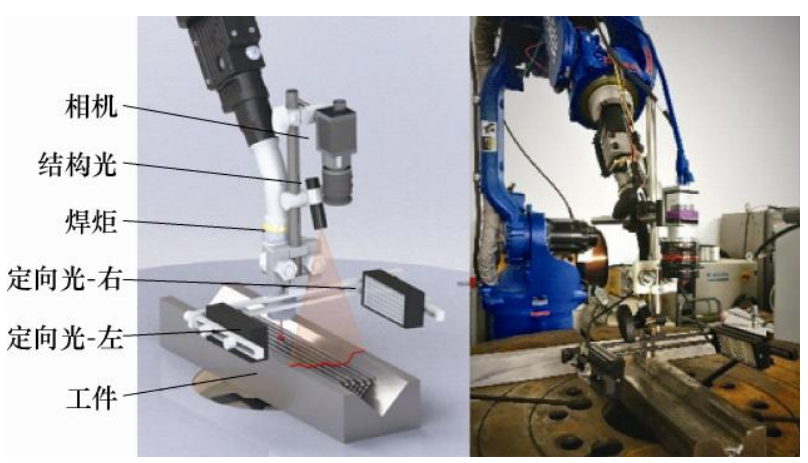

图 1 多层多道熔丝焊接试验系统

电子束熔丝沉积增材制造试验装置如图 2 所 示, 包括: 真空电子束熔丝沉积系统, 多轴数控 运动系统, 钛合金增材制造工件, 线结构光源, 左右对称的定向光源，用于产生同步触发信号波 形的 PLC，千兆以太网工业相机，计算机图像处 理系统等。

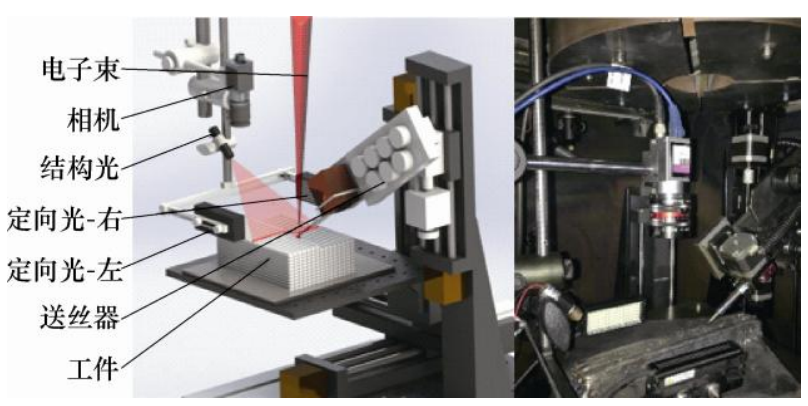

图 2 电子束熔丝沉积增材制造试验系统

2 定向光影视觉信息特征提取

图 3 展示了定向光入射至多道焊缝表面的状 况。由于表面张力的作用, 焊缝往往呈现微上凸状。 因此, 当定向光从侧面入射焊缝区域时，同一焊道 两侧的辐照度存在微小差异。根据几何光学关系可 知, 当左侧定向光以 $\beta$ 的投射角度(定向光与母材的

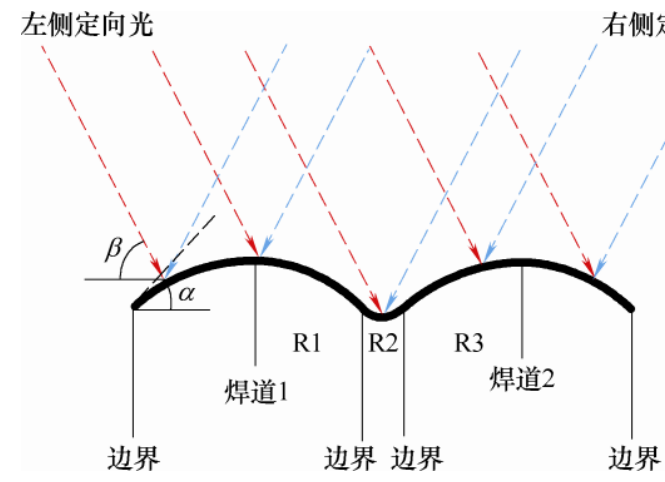

图 3 定向光线入射至多道焊缝表面示意图 
夹角)入射至根部焊趾角为 $\alpha$ 的焊道表面时, 焊道左 右两侧的辐照度之比近似为

$$
\frac{P_{\mathrm{L}}}{P_{\mathrm{R}}} \approx \frac{\sin (\beta+\alpha)}{\sin (\beta-\alpha)}>1
$$

式中, $P_{\mathrm{L}}$ 为焊道左侧辐照度; $P_{\mathrm{R}}$ 为焊道右侧辐照度。

即焊道左侧的辐照度大于右侧。同理, 当右侧 定向光点亮时, 焊道左侧的辐照度小于右侧。但是, 在相邻焊道边界区域处, 辐照度分布情况略有不同。 相邻焊道间的熔合线较为平缓, 无论是左侧定向光 入射还是右侧定向光入射, 相邻焊道边界处的辐照 度基本相当。

综合以上分析可知, 图 3 的三个区域 $\mathrm{R} 1, \mathrm{R} 2$ 和 R3 在左右两侧定向光入射时呈现不同的辐照度 分布情况。当左侧定向光入射时, 区域 $R 1$ 的辐照 度低于区域 $R 3$; 当右侧定向光入射时, 区域 $R 1$ 的 辐照度高于区域 R3; 无论是左侧定向光入射还是右 侧定向光入射, 区域 R2 的辐照度基本相当。因此, 若将两个定向光源单独点亮时获得的焊缝灰度图像 作差, 则在焊道边界附近会呈现 “高灰度-低灰度高灰度” 的过渡区域。

图 4 上部分展示了双侧光影图像的作差结果, 在焊道边界附近确实存在 “高灰度-低灰度-高灰度” 的过渡区域, 验证了以上的分析结果。实际上, 焊 缝表面的高度分布是极不规则的, 当左侧(右侧)定 向光源点亮时, 图像的某些区域可能会出现区域 R1 灰度高于(低于)区域 R3 的现象, 但只要焊道存在一 定的微小凸起, 使得区域 R1 和区域 R3 处的辐照度 存在差异, 那么这两个区域就可能存在较为明显的

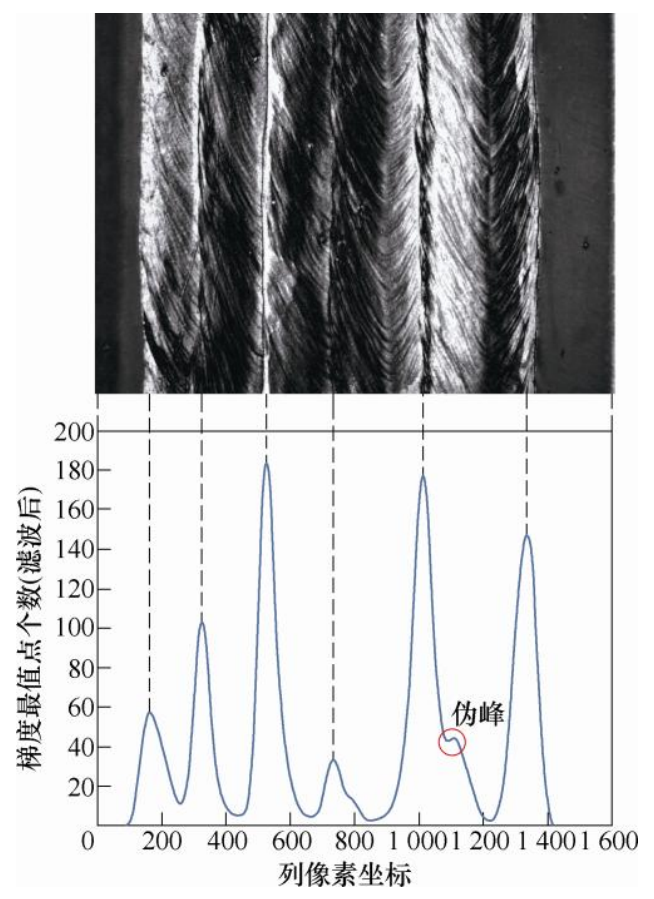

图 4 焊缝双侧光影图像作差结果及灰度梯度分布
灰度差异, 双侧光影图像作差后仍然会呈现 “高灰 度-低灰度-高灰度” 的过渡区域。图 4 下部分是利 用 Sobel 算子计算的灰度梯度分布。

\section{3 结构光传感信息提取}

首先对结构光条图像进行预处理, 通过阈值分 割方法提取光条中心位置, 获得单像素宽度的光条 曲线如图 5 所示。图 6 展示了利用 Savitzky-Golay 滤波器计算的二阶导数结果。进一步以二阶导数曲 线的中值作为阈值, 对二阶导数计算结果进行阈值 分割, 并提取其中的正极大值点位置, 获得母材与 焊道边界以及相邻焊道边界的候选点位置, 如图 7 所示, 灰点位置对应候选点提取结果, 候选点数量 为 45 个。由此可见, 母材与焊道的边界以及相邻焊 道的边界确实对应着二阶导数的正极大值点, 但仅 凭该特征还不足以准确提取焊道边界位置。此外, 根据结构光传感信息也无法判断当前焊道的数量。

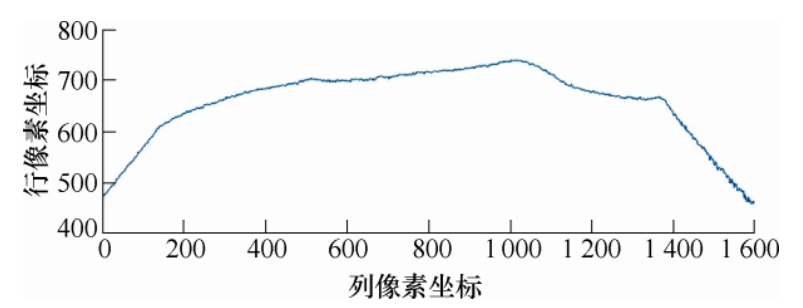

图 5 结构光光条图像预处理结果

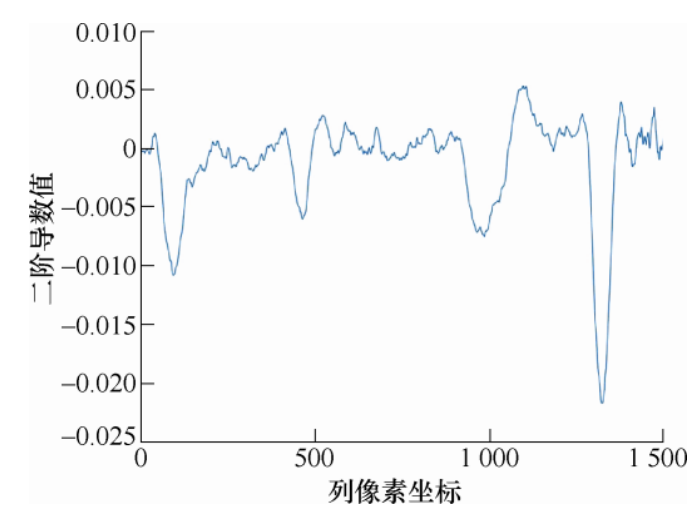

图 6 Savitzky-Golay 滤波器计算的二阶导数值

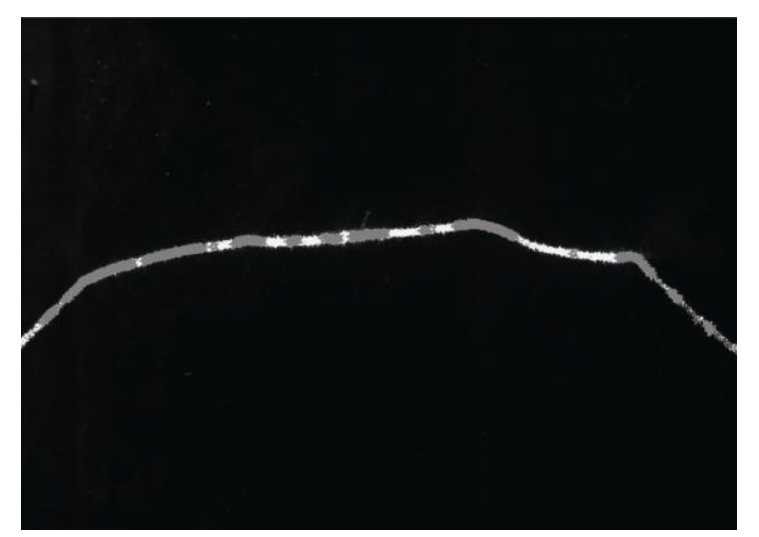

图 7 阈值分割后获得的边界候选点 


\section{4 基于信息融合的图像处理}

弧焊过程和电子束增材制造过程中的飞溅等因 素严重影响成像质量, 而且飞溅的空间分布往往是 不均匀且时变的。对相邻帧图像进行适当的时域融 合处理，有望消除飞溅等因素的干扰。设 $t$ 时刻相 机获得的图像为 $I_{0, t}(x, y)$, 选取时域相邻的 $n$ 帧图像 对应像素点的灰度最小值构成新图像, 以消除弧光、 飞溅等干扰，即式(1)

$$
I_{t}(x, y)=\min \left\{I_{0, t}(x, y), I_{0, t-1}(x, y), \cdots, I_{0, t-(n-1)}(x, y)\right\}
$$

考虑到工件相对焊炬运动, 因此时域相邻帧图 像的视场并非完全重合。若式(1)的图像数量 $n$ 过大, 则会造成图像整体灰度偏低, 甚至容易丢失重要的 视觉特征信息。若对时域相邻帧图像进行配准后再 进行式(1)的融合操作, 势必会牺牲实时性。

对于定向光源照射下的图像, 即使 $n=2$ 也会使 图像灰度严重偏低, 容易掩盖有效的特征信息。这 是因为焊缝区域的灰度空间分布是十分不均匀的, 高灰度区域附近可能分布着低灰度区域, 反之亦然; 而焊接过程中工件与焊炬的相对运动可能使相邻帧 图像的高灰度区域与低灰度区域重叠, 导致融合处 理后图像整体灰度水平严重下降。为能够在消除弧 光、飞溅干扰的同时保持图像的整体灰度水平, 首 先利用式(1)取 $n=2$ 进行融合处理, 以获得新图像 $I_{1, t}(x, y)$; 再取 $I_{1, t}(x, y)$ 和 $I_{1, t-1}(x, y)$ 对应像素点灰度值 的最大值构成最终的图像 $I_{t}(x, y)$, 即式(2)

$$
\begin{gathered}
I_{t}(x, y)=\max \left\{I_{1, t}(x, y), I_{1, t-1}(x, y)\right\}= \\
\max \left\{\min \left\{I_{0, t}(x, y), I_{0, t-1}(x, y)\right\},\right. \\
\left.\min \left\{I_{0, t-1}(x, y), I_{0, t-2}(x, y)\right\}\right\}
\end{gathered}
$$

图 8 展示了经两步融合方法处理后的结果, 焊

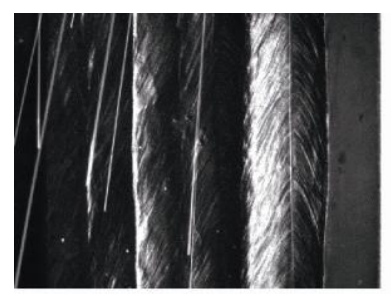

(a) $t-2$ 时刻图像

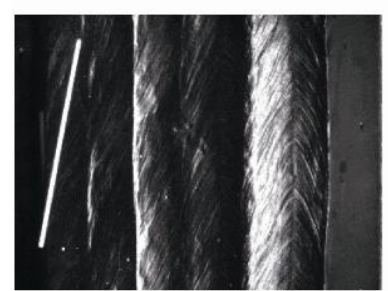

(c) $t$ 时刻图像

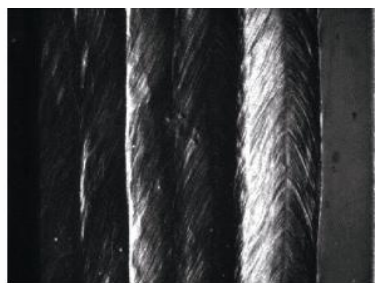

(b) $t-1$ 时刻图像

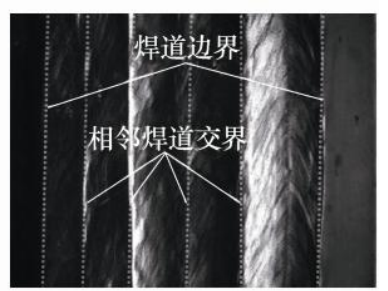

(d) 时域融合处理后图像
图 8 定向光图像的时域融合处理
接过程的飞溅等干扰因素已基本消除，且仍能保持 原图像的整体灰度水平。

对于线结构光图像，则直接采用式(1)取 $n=3$ 消 除弧光和飞溅干扰, 融合处理结果如图 9 所示, 其 中 $t-2$ 时刻图像有较为强烈的飞溅干扰, $t$ 时刻图像 存在的弧光干扰使背景灰度上升。经时域融合处理 后，弧光和飞溅干扰获得消除。

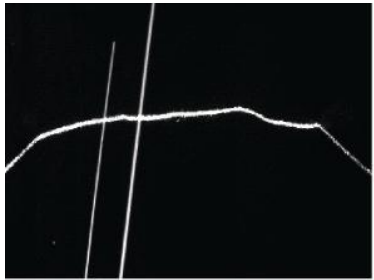

(a) $t-2$ 时刻图像

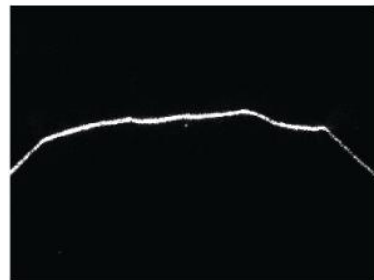

(c) $t$ 时刻图像 (b) $t-1$ 时刻图像

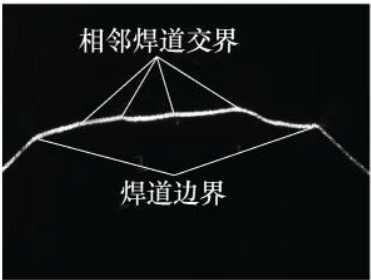

(d) 时域融合处理后图像

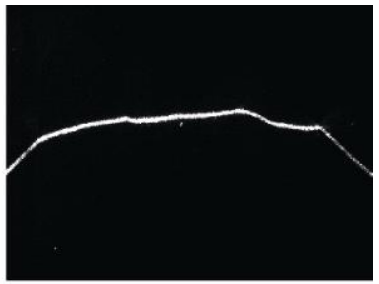

图 9 结构光图像的时域融合处理

定向光影特征既可获得焊道数量也可获得边界 的大体位置, 但与真实边界位置可能还存在一定的 偏差; 结构光传感信息可反映焊道截面的三维信息, 亦能根据激光光条曲线的二阶导数值预估边界位 置, 但预估的准确性欠佳, 且候选点数量往往较大。 综合以上分析结果，提出一种基于定向光影特征和 结构光传感信息融合的多道焊接轨迹识别算法, 流 程如图 10 所示。

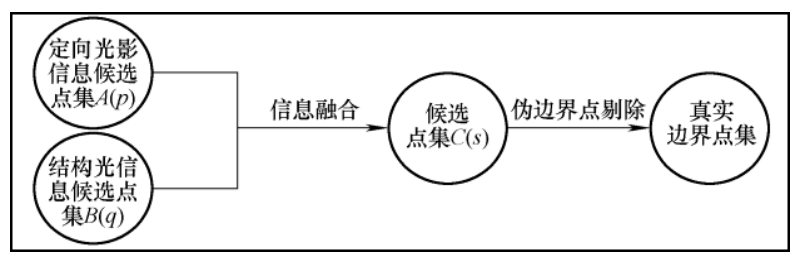

图 10 基于定向光影特征和结构光传感信息融合的 焊道轨迹识别算法流程

采用 “多光源交替频闪-相机同步采集” 方法实 现多视觉特征信息的近同步获取。使三个光源(定向 面光源 1 、定向面光源 2 和线结构光光源) 以一定频 率交替点亮，并使相机同步采集每个光源单独点亮 时的图像。

\section{5 视觉检测试验结果分析}

利用图 1 所示多层多道弧焊系统开展试验。试 
件表面可见 5 道焊缝, 其中, 第 2 道位于第六层, 第 3 道位于第五层。采用熔化极氩气保护焊工艺, 焊接电流为 $220 \mathrm{~A}$, 焊接速度为 $240 \mathrm{~mm} / \mathrm{min}$, 相机 帧频设置为 30 帧/s, 图像大小为 1600 像素 $\times 1200$ 像素, 视场大小约为 $33 \mathrm{~mm} \times 25 \mathrm{~mm}$, 分辨率约为 $0.02 \mathrm{~mm} /$ 像素。

利用图 2 所示系统开展试验, 对电子束熔丝沉 积增材制造过程的已沉积钛合金层进行三维重建。 试验采用 $60 \mathrm{kV}$ 加速电压, 束流为 $140 \mathrm{~mA}$, 平台移 动速度为 $500 \mathrm{~mm} / \mathrm{min}$ 。图像参数与上组试验相同。

在熔丝制造过程中, 采用 “多光源交替频闪相机同步采集” 方法, 以触发信号同步相机和各个 光源间的时序关系; 以时域融合方法消除弧光和飞 溅干扰, 对原始图像进行预处理; 基于定向光影特 征和结构光传感信息融合的空域处理方法提取焊道 轨迹位置。

试验结果表明, 在熔丝制造过程中, “多光源交 替频闪-相机同步采集”系统经受实际工况环境考验, 保持正常状态; 经时空域融合算法处理后, 坡口侧壁 与焊缝问的边界以及相邻焊道间的边界能较好地识 别出来, 单次图像处理耗时不超过 $30 \mathrm{~ms}(2.3 \mathrm{GHz}$ CPU, 4 GB RAM 工业计算机), 能满足焊接过程实 时检测需求; 最大检测偏差控制在 $0.6 \mathrm{~mm}$ 以内。电 弧多层多道焊缝形貌三维重构如图 11 所示, 从图中 可观察到清晰的 2 条焊道边界和 4 条相邻焊道交界, 焊后工件表面呈典型 $\mathrm{V}$ 形坡口多层多道焊形貌。电 子束熔丝沉积增材制造已沉积层三维重构结果如图 12 所示, 从图中可观察到 2 条焊道边界和 6 条相邻 焊道交界, 沉积层表面呈典型熔丝沉积波浪状形貌, 焊道交界处为波谷, 焊道中部为波峰, 工件两侧呈 圆弧下塌状。实测当焊道中心余高在 $1 \mathrm{~mm}$ 以上时, 焊道两侧辐照度差异明显, 可准确获取焊道交界位 置。当余高在 $0.3 \mathrm{~mm}$ 以下时, 焊道两侧辐照度差异 变得不再明显, 无法获得焊道交界位置。

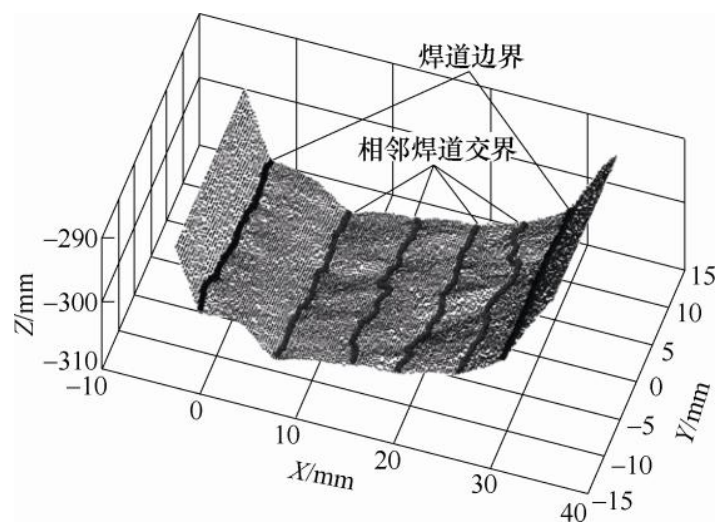

图 11 多道弧焊过程焊缝形貌三维重建和路径识别

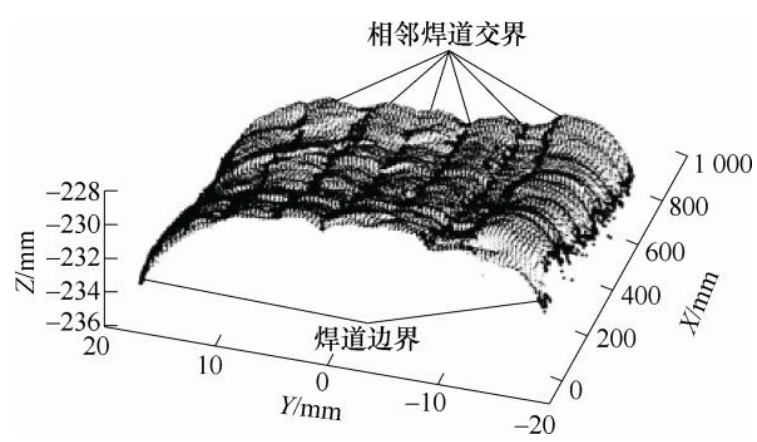

图 12 电子束熔丝增材制造沉积层形貌三维重构

\section{6 结论}

（1）本文提出了一种人为构造定向光照补光方 法, 在该条件下焊缝边界区的灰度明显高于母材区, 提高了后续图像处理的识别成功率。

（2）针对电弧熔丝多层多道焊接和电子束熔丝 沉积增材制造两种使用环境, 采用 “双光源交替频 闪-相机同步采集” 方案实现了焊缝双侧光影特征图 像的近同步获取。

（3）提出了基于定向光影视觉信息与结构光传 感信息的实时融合处理算法, 实现了实时提取焊缝 轨迹信息, 可适用于余高低至 $1 \mathrm{~mm}$ 的成形焊缝轨 迹实时检测。为实现机器人多层多道电弧焊接和熔 丝沉积增材制造过程的路径在线识别及实时自动导 引提供了新的技术途径。

\section{参 考 文 献}

[1] DING Donghong, PAN Zengxi, CUIURI D, et al. Wire-feed additive manufacturing of metal components: Technologies, developments and future interests[J]. The International Journal of Advanced Manufacturing Technology, 2015, 81: 465-481.

[2] TAMINGER K M, HARFLEY R A. Electron beam freeform fabrication : A rapid metal deposition process $[\mathrm{C} / \mathrm{CD}] / /$ Society of Plastics Engineers. Proceedings of the 3rd Annual Automotive Composites Conference, Sep. 9-10, 2003, NASA Technical Reports Server, 2003.

[3] GOCKEL J, BEUTH J, TAMINGER K M. Integrated control of solidification microstructure and melt pool dimensions in electron beam wire feed additive manufacturing of Ti-6Al-4V[J]. Additive Manufacturing, 2014, 1: 119-126.

[4] ZHANG Binggang, ZHAO Jian, LI Xiaopeng, et al. Effects of filler wire on residual stress in electron beam 
welded QCr0.8 copper alloy to 304 stainless steel joints[J]. Applied Thermal Engineering, 2015, 80: 261-268.

[5] WANG T, ZHANG Y, LI X, et al. Influence of beam current on microstructures and mechanical properties of electron beam welding-brazed aluminum-steel joints with an Al5Si filler wire[J]. Vacuum, 2017, 141: 281-287.

[6] NIE Z, WANG G, MCGUFFIN-CAWLEY J D, et al. Experimental study and modeling of $\mathrm{H} 13$ steel deposition using laser hot-wire additive manufacturing $[\mathrm{J}]$. Journal of Materials Processing Technology, 2016, 235: 171-186.

[7] DEMIR A G. Micro laser metal wire deposition for additive manufacturing of thin-walled structures[J]. Optics and Lasers in Engineering, 2018， 100: 9-17.

[8] LIU W, LIU S, MA J, et al. Real-time monitoring of the laser hot-wire welding process[J]. Optics \& Laser Technology, 2014, 57: 66-76.

[9] WANG $\mathrm{F}$, WILLIAMS $\mathrm{S}$, COLEGROVE $\mathrm{P}$, et al. Microstructure and mechanical properties of wire and arc additive manufactured Ti-6Al-4V[J]. Metallurgical and Materials Transactions A, 2013，44(2): 968-977.

[10] 都东, 邹怡蓉, 常保华. 焊接视觉传感及自动跟踪技术 的现状与发展[J]. 航空制造技术，2010(9): 40-42.

DU Dong, ZOU Yirong, CHANG Baohua. Current status and development of welding vision sensing and automatic tracking technology[J]. Aviation Manufacturing Technology, 2010(9): 40-42.

[11] 邹怡蓉, 曾锦乐, 都东. 可视化焊接的发展 $[\mathrm{J}]$. 电焊机, 2013(5): 17-19.

ZOU Yirong, ZENG Jinle, DU Dong. Development of visual welding[J]. Electric Welder, 2013(5): 17-19.

[12] 蒋凡, 李元锋, 陈树君. 焊接电弧监测技术研究现状及 展望[J]. 机械工程学报，2018，54(2)：16-26.

JIANG Fan, LI Yuanfeng, CHEN Shujun. Review of welding arc monitoring technology[J]. Journal of Mechanical Engineering, 2018，54(2): 16-26.

[13] 邹炎謎, 周卫林, 王研博. 基于概率连续模型的激光视 觉焊缝自动跟踪 $[\mathrm{J}]$. 机械工程学报, 2017, 53(10): 70-78.

ZOU Yanbiao, ZHOU Weilin, WANG Yanbo. Automatic laser visual seam tracking based on probabilistic continuous model[J]. Journal of Mechanical Engineering, 2017, 53(10): 70-78.

[14] ZENG J, CHANG B, DU D, et al. A precise visual method for narrow butt detection in specular reflection workpiece welding[J]. Sensors, 2016， 16(9): 1480.
[15] ZENG J, CHANG B, DU D, et al. A vision-aided 3D path teaching method before narrow butt joint welding[J]. Sensors, 2017, 17(5): 1099

[16] ZENG J, CHANG B, DU D, et al. A visual weld edge recognition method based on light and shadow feature construction using directional lighting[J]. Journal of Manufacturing Processes, 2016, 24: 19-30.

[17] ZENG J, CHANG B, DU D, et al. A weld position recognition method based on directional and structured light information fusion in multi-Layer/multi-pass welding[J]. Sensors, 2018, 18(1): 129.

[18] SHAO J, DU D, CHANG B, et al. Automatic weld defect detection based on potential defect tracking in real-time radiographic image sequence[J]. NDT \& E International, 2012, 46: 14-21.

[19] SHAO J, DU D, SHI H, et al. Automatic weld recognition and extraction from real-time X-ray images using quadratic curve fitting and multi-order differences analysis of intensity profile[J]. Insight-Non-Destructive Testing and Condition Monitoring, 2011, 53(10): 562-569.

[20] DU D, HOU R, SHAO J, et al. Registration of real-time $\mathrm{X}$-ray image sequences for weld inspection[J]. Nondestructive Testing and Evaluation, 2010, 25(2): 153-159.

[21] ZOU Y, DU D, CHANG B, et al. Automatic weld defect detection method based on Kalman filtering for real-time radiographic inspection of spiral pipe[J]. NDT \& E International, 2015, 72: 1-9.

[22] 都东, 王胜华, 王力, 等. 基于对象特征分析的焊缝识 别视觉传感技术[J]. 焊接学报, 2008, 29(10): 108-112. DU Dong, WANG Shenghua, WANG Li, et al. Vision-sensing technology for weld seam recognition based on object feature analysis[J]. Journal of Welding, 2008, 29(10): 108-112.

[23] 邹怡蓉, 都东, 曾锦乐, 等. 基于多视觉特征获取与信 息融合的焊道识别方法 [J]. 焊接学报, 2013, 34(5): 33-36.

ZOU Yirong, DU Dong, ZENG Jinle, et al. A method of bead identification based on multi-visual feature acquisition and information fusion[J]. Journal of Welding, 2013, 34(5): 33-36.

[24] 邹怡容, 吴哲明, 曾凯, 等. 基于焊缝纹理特征的视觉 识别方法 [J]. 焊接学报, 2009, 30(5): 13-16.

ZOU Yirong, WU Zheming, ZENG Kai, et al. A visual recognition method based on weld texture features[J]. Journal of Welding, 2009, 30(5): 13-16. 
[25] 邹怡蓉, 吴哲明, 郭桂林, 等. 基于图像色彩信息的焊 缝识别算法[J]. 焊接学报, 2009, 30(10): 37-40.

ZOU Yirong, WU Zheming, GUO Guilin, et al. Weld seam recognition algorithm based on image color information[J]. Journal of Welding, 2009, 30(10): 37-40.
作者简介: 常树鹤, 男, 1993 年出生, 博士研究生。主要研究方向为电 子束熔丝沉积增材制造过程智能控制。

E-mail: changsh15@mails.tsinghua.edu.cn

都东(通信作者), 男, 1962 年出生, 博士, 长聘教授。主要研究方向为 机器人与智能成形制造。

E-mail: dudong@tsinghua.edu.cn 